\title{
ON THE STOCHASTIC REGULARITY OF SEQUENCE TRANSFORMATIONS OPERATING IN A BANACH SPACE
}

I. Introduction. In numerical analysis, convergence acceleration methods have been studied for many years and applied to various situations (see [3]).

On the other hand, the jacknife, a well-known statistical procedure for bias reduction, has been studied in the recent years by several authors (for example, see [4]), who established a direct parallel between the jacknife statistic and the $e_{n}$-transformation which is a sequence transformation used in numerical analysis for accelerating the convergence of a sequence by extrapolation.

Thus the idea of applying sequence transformations studied in numerical analysis to sequences of random elements converging in a stochastic mode was born.

In this paper, we define a new notion of stochastic regularity and we develop linear transformations called summation processes applied to sequences of random elements in a Banach space. It is shown that the regular summation process defined in numerical analysis is not always regular for every mode of stochastic convergence.

\section{Definitions and notations}

II.1. Definitions relating to numerical analysis. Let $E$ be a Banach space with norm \|\|$_{E}$, and let $\mathcal{S}(E)$ be the set of sequences whose terms are elements of $E$.

Let $T$ be a transformation which transforms a sequence $\left(S_{n}\right) \in \mathcal{S}(E)$ into another sequence $\left(T_{n}\right) \in \mathcal{S}(E)$. We say that $T$ operates in $\mathcal{S}(E)$. Let

1991 Mathematics Subject Classification: 65U05, 65B99.

Key words and phrases: summation processes, stochastic convergence, regularity. 
$\left(S_{n}\right)$ converge to $S$. Then, if $\left(T_{n}\right)$ also converges to $S$, we say that $T$ is regular for the sequence $\left(S_{n}\right)$.

If, for each converging sequence $\left(S_{n}\right) \in \mathcal{S}(E),\left(T_{n}\right)$ also converges to the same limit, we say that $T$ is regular for $\mathcal{S}(E)$.

II.2. Definitions relating to probability

II.2.1. Let $(\Omega, \mathcal{A}, P)$ be a probability space and let $F$ be a separable Banach space with norm \|\|$_{F}$ and $\sigma$-field $\mathcal{B}$ of Borel sets. Let $S$ be a measurable mapping from $(\Omega, \mathcal{A})$ into $(F, \mathcal{B})$; we call it an $F$-valued random element. Let $\left(S_{n}\right)$ be a sequence of $F$-valued random elements (defined on the same field $\Omega$ ); under the assumption of separability, it is known that $\left\|S_{n}-S\right\|_{F}$ is a random variable defined on $\Omega$ (see [1] and [5]).

II.2.2. Now, we recall the definitions of stochastic convergences.

(a) Convergence in distribution:

$S_{n} \stackrel{\mathcal{D}}{\rightarrow} S \Leftrightarrow$ for all $B \in \mathcal{B}$ with $P(S \in \partial B)=0, P\left(S_{n} \in B\right) \rightarrow P(S \in B)$.

(b) Convergence in probability:

$$
S_{n} \stackrel{P}{\rightarrow} S \Leftrightarrow \forall \varepsilon>0, P\left(\left\|S_{n}-S\right\|_{F} \geq \varepsilon\right) \rightarrow 0 .
$$

(c) Almost sure convergence:

$$
S_{n} \stackrel{\text { a.s. }}{\rightarrow} S \Leftrightarrow P\left(\left\|S_{n}-S\right\|_{F} \rightarrow 0\right)=1 .
$$

(d) Almost complete convergence:

$$
S_{n} \stackrel{\text { a.c. }}{\rightarrow} S \Leftrightarrow \forall \varepsilon>0, \quad \sum_{n \geq 1} P\left(\left\|S_{n}-S\right\|_{F} \geq \varepsilon\right)<\infty .
$$

(e) Convergence in the rth mean:

$$
S_{n} \stackrel{N_{r}}{\rightarrow} S \Leftrightarrow E\left(\left\|S_{n}-S\right\|_{F}\right)^{r} \rightarrow 0 .
$$

Let us recall the following implications:

$$
\text { a.c. } \Rightarrow \text { a.s. } \Rightarrow P \Rightarrow \mathcal{D} \quad \text { and } \quad N_{r} \Rightarrow N_{r^{\prime}} \Rightarrow P, \quad \text { with } \quad r^{\prime}<r
$$

(for the proof, see [2]).

II.2.3. Finally, we set:

(a) $\mathcal{L}_{0}(\Omega, \mathcal{A}, P, F)$ or $\mathcal{L}_{0}(P, F)$, the vector space of $F$-valued random elements, and $L_{0}(P, F)=\mathcal{L}_{0}(P, F) / \sim$, the quotient space by the equivalence relation " $S=T$ almost surely".

(b) $\mathcal{L}_{r}(P, F)$, the vector subspace of $\mathcal{L}_{0}(P, F)$ defined by $S \in \mathcal{L}_{r}(P, F)$ iff $\int_{\Omega}\|S\|_{F}^{r} d P<\infty$, and $L_{r}(P, F)=\mathcal{L}_{r}(P, F) / \sim$. It is known that for $r>0$, $L_{r}(P, F)$ is a Banach space with norm $\|S\|_{L_{r}}=\left(\int_{\Omega}\|S\|_{F}^{r} d P\right)^{1 / r}$ associated with the convergence $S_{n} \stackrel{N_{r}}{\rightarrow} S$. 
(c) $\mathcal{L}_{\infty}(P, F)$, the vector space of $F$-valued random elements $S$ such that $\|S\|_{F}<\infty$ and $L_{\infty}(P, F)=\mathcal{L}_{\infty}(P, F) / \sim$. It is known that $L_{\infty}(P, F)$ is a Banach space with norm $\|S\|_{L_{\infty}}=\operatorname{ess} \sup \|S\|_{F}$ (see [5]). (We write $S_{n} \stackrel{N_{\infty}}{\longrightarrow} S \Leftrightarrow\left\|S_{n}-S\right\|_{L_{\infty}} \rightarrow 0$.)

\section{Stochastic regularity of a sequence transformation}

III.1. General case. Let $\mathcal{S}\left[\mathcal{L}_{0}(P, F)\right]$ be the set of sequences of $F$-valued random elements and let $\left(S_{n}\right)$ be a sequence converging to $S \in \mathcal{L}_{0}(P, F)$ for one of the modes $\mathcal{M}$ defined in II.2.

Let $T$ be a sequence transformation operating in $F$.

Taking $s_{n}=S_{n}(\omega)$ and $t_{n}=T_{n}(\omega)$, for $\omega \in \Omega$, if $T_{n}$ is measurable for all $n$ (which we suppose), we may consider that $T$ operates in $\mathcal{L}_{0}(P, F)$ and transforms the sequence $\left(S_{n}\right)$ into another sequence $\left(T_{n}\right)$.

Definition III.1. We say that $T$ is $\mathcal{M}$-regular for $\left(S_{n}\right)$ if $S_{n} \stackrel{\mathcal{M}}{\rightarrow} S$ implies $T_{n} \stackrel{\mathcal{M}}{\rightarrow} S$.

Definition III.2. We say that $T$ is $\mathcal{M}$-regular for $\mathcal{S}\left[\mathcal{L}_{0}(P, F)\right]$ if for every sequence $\left(S_{n}\right)$ of $F$-valued elements such that $S_{n} \stackrel{\mathcal{M}}{\rightarrow} S$ we have $T_{n} \stackrel{\mathcal{M}}{\rightarrow} S$.

R e m a r k. From the connections between the different modes of convergence, we immediately have the corresponding implications:

$$
\begin{gathered}
T \text { a.c.-regular } \Rightarrow T \text { a.s-regular } \Rightarrow T P \text {-regular } \Rightarrow T \mathcal{D} \text {-regular, } \\
T N_{r} \text {-regular } \Rightarrow T N_{r^{\prime}} \text {-regular with } r^{\prime}<r .
\end{gathered}
$$

This obviously holds for a sequence $\left(S_{n}\right)$ well defined under the condition that $\left(S_{n}\right)$ converges for the mode $\mathcal{M}$ concerned. Applying the definition of almost sure convergence, we obtain

THEOREM III.1. If $T$ is regular for $\mathcal{S}(F)$, then $T$ is a.s.-regular for $\mathcal{S}\left[\mathcal{L}_{0}(P, F)\right]$.

III.2. Finite summation process. We call so the simplest linear transformation defined by $\left(a_{0}, \ldots, a_{k}\right) \in \mathbb{R}^{k}$ or $\mathbb{C}^{k}$, with $k$ fixed in $\mathbb{N}$. $\left(S_{n}\right)$ being a sequence in $\mathcal{S}(E)$, the sequence $\left(T_{n}\right)$ is defined by $T_{n}=a_{0} S_{n}+\ldots+a_{k} S_{n+k}$.

Such a process is said to be regular if $a_{0}+a_{1}+\ldots+a_{k}=1$. Under this assumption, clearly, for any Banach space $E$, the associated transformation operating in $\mathcal{S}(E)$ is regular for $\mathcal{S}(E)$.

Now, let $\left(S_{n}\right) \in \mathcal{S}\left[\mathcal{L}_{0}(P, F)\right]$. From Theorem III.1, we obviously have the following result concerning almost sure convergence: 
THEOREM III.2. Let $\left(S_{n}\right)$ be a sequence of F-valued elements and $T$ the preceding transformation operating in $\mathcal{S}\left[\mathcal{L}_{0}(P, F)\right]$. If the process is regular, then $T$ is a.s.-regular for $\mathcal{S}\left[\mathcal{L}_{0}(P, F)\right]$.

Now, taking $E=L_{r}(P, F)$ with norm \|\|$_{L_{r}}$, we obtain the result concerning convergence in the $r$ th mean for all $r \in] 0, \infty[$ and convergence in $L_{\infty}(P, F)$ :

THEOREM III.3. Under the assumptions of Theorem III.2, $T$ is $N_{r}$ regular for $\mathcal{S}\left[\mathcal{L}_{r}(P, F)\right]$ for all $\left.\left.r \in\right] 0, \infty\right]$.

Concerning almost complete convergence, we have

ThEOREM III.4. Under the assumptions of Theorem III.2, $T$ is a.c.regular for $\mathcal{S}\left[\mathcal{L}_{0}(P, F)\right]$.

Proof. Suppose that, for all $\varepsilon>0, \sum_{n \in \mathbb{N}} P\left(\left\|S_{n}-S\right\|_{F} \geq \varepsilon\right)<\infty$.

Since $T_{n}-S=a_{0}\left(S_{n}-S\right)+\ldots+a_{k}\left(S_{n+k}-S\right)$, we have

$$
\left\|T_{n}-S\right\|_{F} \leq\left|a_{0}\right| \cdot\left\|S_{n}-S\right\|_{F}+\ldots+\left|a_{k}\right| \cdot\left\|S_{n+k}-S\right\|_{F} .
$$

Now, $\left\|S_{j}-S\right\|_{F}<\varepsilon$ for all $j \in\{n, \ldots, n+k\}$ implies

$$
\left\|T_{n}-S\right\|_{F}<\varepsilon\left(\left|a_{0}\right|+\ldots+\left|a_{k}\right|\right)=M \varepsilon
$$

where $M$ is a constant. Hence

$$
P\left(\bigcap_{j=n}^{n+k}\left\|S_{j}-S\right\|_{F}<\varepsilon\right) \leq P\left(\left\|T_{n}-S\right\|_{F}<M \varepsilon\right) .
$$

It follows that

and

$$
P\left(\bigcup_{j=n}^{n+k}\left\|S_{j}-S\right\|_{F} \geq \varepsilon\right) \geq P\left(\left\|T_{n}-S\right\|_{F} \geq M \varepsilon\right)
$$

$$
P\left(\left\|T_{n}-S\right\|_{F} \geq M \varepsilon\right) \leq \sum_{j=n}^{n+k} P\left(\left\|S_{j}-S\right\|_{F} \geq \varepsilon\right) .
$$

Writing this inequality for $n=0,1, \ldots$ and summing we obtain

$$
\sum_{n \in \mathbb{N}} P\left(\left\|T_{n}-S\right\|_{F} \geq M \varepsilon\right) \leq(k+1) \sum_{n \in \mathbb{N}} P\left(\left\|S_{n}-S\right\|_{F} \geq \varepsilon\right) .
$$

Taking $\varepsilon=\varepsilon^{\prime} / M$, for any $\varepsilon^{\prime}>0$ we obtain $\sum_{n \in \mathbb{N}} P\left(\left\|T_{n}-S\right\|_{F} \geq \varepsilon^{\prime}\right)<\infty$.

Concerning convergence in probability, we have

THEOREM III.5. Under the assumptions of Theorem III.2, $T$ is P-regular for $\mathcal{S}\left[\mathcal{L}_{0}(P, F)\right]$.

Proof. Suppose that, for all $\varepsilon>0, P\left(\left\|S_{n}-S\right\|_{F} \geq \varepsilon\right) \rightarrow 0$. We may write (1). Then, for each $\varepsilon>0,\left\|T_{n}-S\right\|_{F} \geq \varepsilon$ implies $\left|a_{0}\right| \cdot\left\|S_{n}-S\right\|_{F}+$ $\ldots+\left|a_{k}\right| \cdot\left\|S_{n+k}-S\right\|_{F} \geq \varepsilon$. Hence 
(2) $\quad P\left\{\left\|T_{n}-S\right\|_{F} \geq \varepsilon\right\}$

$$
\leq P\left\{\left|a_{0}\right| \cdot\left\|S_{n}-S\right\|_{F}+\ldots+\left|a_{k}\right| \cdot\left\|S_{n+k}-S\right\|_{F} \geq \varepsilon\right\} .
$$

But we know that the convergence in probability of random variables is compatible with the vector space structure of $\mathbb{R}$. Then $\left\|S_{n}-S\right\|_{F} \stackrel{P}{\rightarrow} 0$ implies $\left|a_{0}\right| \cdot\left\|S_{n}-S\right\|_{F}+\ldots+\left|a_{k}\right| \cdot\left\|S_{n+k}-S\right\|_{F} \stackrel{P}{\rightarrow} 0$ and the result comes from (2).

R e mark. Concerning convergence in distribution, the following example proves that we do not obtain a similar result.

Take $k=1, a_{0}=a_{1}=1 / 2$ and $F=\mathbb{R}$. The sequence $\left(S_{n}\right)$ is defined by

$$
S_{2 n}=S_{0}, \quad S_{2 n+1}=-S_{0} \quad \text { for } n \in \mathbb{N} .
$$

Suppose now that $S_{0}$ has a symmetric distribution different from the Dirac measure $\delta_{0}$ (that is, $P\left\{S_{0} \neq 0\right\}>0$ ). It follows that $S_{n}$ has the same distribution as $S_{0}$, hence $S_{n} \stackrel{\mathcal{D}}{\rightarrow} S_{0}$. But $T_{n}=0$, for all $n$, and thus $T_{n} \stackrel{\mathcal{D}}{\leftrightarrow} S_{0}$.

III.3. Summation process. A summation process is the linear transformation defined by an infinite triangular matrix $A=\left(a_{k}^{j}\right)_{k \in \mathbb{N}, 0 \leq j \leq k}$ where the $a_{k}^{j}$, s are constants of $\mathbb{C}$ or $\mathbb{R}$. It transforms a sequence $\left(s_{n}\right) \in \mathcal{S}(F)$ into the sequence $\left(t_{k}^{(n)}\right)$ defined by $t_{k}^{(n)}=a_{k}^{0} s_{n}+\ldots+a_{k}^{k} s_{n+k}$, and a sequence $\left(S_{n}\right) \in \mathcal{S}\left[\mathcal{L}_{0}(p, F)\right]$ into the sequence $\left(T_{k}^{(n)}\right)$ with $T_{k}^{(n)}(\omega)=t_{k}^{(n)}$ for $\omega \in \Omega$.

Such a transformation is said to be regular (or $A$ is regular) if it satisfies the assumptions

(i) $\sum_{j=0}^{k}\left|a_{k}^{j}\right| \leq M$ for all $k \in \mathbb{N}$,

(ii) $\lim _{k \rightarrow \infty} a_{k}^{j}=0$ for all $j \in \mathbb{N}$,

(iii) $\lim _{k \rightarrow \infty} \sum_{j=0}^{k} a_{k}^{j}=1$.

It is said to be total (or $A$ is total) if (iii) becomes

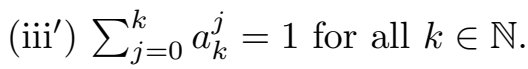

Clearly, in the case of a total process, for each fixed $k$ in $\mathbb{N}$ the transformation $T_{(k)}$ which transforms the sequence $\left(S_{n}\right)$ into the sequence $\left(T_{k}^{(n)}\right)_{n \in \mathbb{N}}$ is a regular finite summation process as studied in II.

In the following, we consider the transformation $T^{(n)}$ which transforms $\left(S_{n}\right)$ into the sequence $\left(T_{k}^{(n)}\right)_{k \in \mathbb{N}}$ with $T_{k}^{(n)}=a_{k}^{0} S_{n}+\ldots+a_{k}^{k} S_{n+k}$, for $n$ fixed. First, let us recall a well known theorem:

TOEPLITZ THEOREM. Let $E$ be a Banach space, $\left(S_{n}\right)$ a sequence in $\mathcal{S}(E)$ and $\left(T_{k}\right)$ the sequence in $\mathcal{S}(E)$ transformed by a summation process $T_{k}=a_{k}^{0} S_{0}+\ldots+a_{k}^{k} S_{k}$. Then a necessary and sufficient condition that, for all converging sequences $\left(S_{n}\right)$, the sequence $\left(T_{k}\right)$ converges to the same limit, is that the process is regular. 
For the proof see [7].

Now, suppose the sequence $\left(S_{n}\right)$ does converge to $S$ for a mode $\mathcal{M}$ of stochastic convergence.

Concerning almost sure convergence, the following result comes from the Toeplitz theorem with $E=F$. For $n$ fixed, it is obvious that the properties of convergence are the same for $T^{(n)}$ and $T^{(0)}$.

THEOREM III.6. Let $\left(S_{n}\right)$ be a sequence of F-valued elements and $T^{(n)}$ ( $n$ fixed) the transformation operating in $\mathcal{S}\left[\mathcal{L}_{0}(P, F)\right]$ associated with a regular summation process. Then $T^{(n)}$ is a.s.-regular for $\mathcal{S}\left[\mathcal{L}_{0}(P, F)\right]$.

Now, taking $E=L_{r}(P, F)$ with norm \|\|$_{L_{r}}$ in the Toeplitz theorem yields a result on convergence in the $r$ th mean, for all $r \in] 0, \infty[$, and convergence in $L_{\infty}(P, F)$.

THEOREM III.7. Under the assumptions of Theorem III.6, $T^{(n)}$ is $N_{r}$ regular for $\mathcal{S}\left[\mathcal{L}_{r}(P, F)\right]$.

Remark 1. Concerning convergence in distribution, the following example proves that we do not obtain $\mathcal{D}$-regularity.

Take $a_{k}^{j}=1 /(k+1)(j=0,1, \ldots, k)$ and $F=\mathbb{R}$. The sequence $\left(S_{n}\right)$ is defined by

$$
S_{2 n}=S_{0}, \quad S_{2 n+1}=-S_{0} \quad \text { for } n \in \mathbb{N},
$$

and suppose that $S_{0}$ has a symmetric distribution different from $\delta_{0}$. Thus $S_{n} \stackrel{\mathcal{D}}{\rightarrow} S_{0}$. But the sequence $\left(T_{k}^{(n)}\right)$ does not converge in distribution to $S_{0}$ since $T_{2 i}^{(n)}=S_{0} /(2 i+1)$ for all $i \in \mathbb{N}$ and $T_{2 i+1}^{(n)}=0$ for all $i \in \mathbb{N}$; hence $T_{k}^{(n)} \stackrel{\text { a.s. }}{\rightarrow} 0$ as $k \rightarrow \infty$.

R e m a r k 2. Concerning convergence in probability, the following example proves that we do not obtain $P$-regularity.

Take $k \in \mathbb{N}^{*}, a_{j}^{k}=1 / k$ for $j=1, \ldots, k$ and $F=\mathbb{R}$. Let $\left(S_{n}\right)_{n \in \mathbb{N}^{*}}$ be a sequence of independent random variables with distribution functions

$$
F_{n}(x)= \begin{cases}1-1 /(x+n) & \text { for } x>0 \\ 0 & \text { for } x \leq 0\end{cases}
$$

Then

$$
\forall \varepsilon>0, P\left(\left|S_{n}\right| \geq \varepsilon\right)=\frac{1}{\varepsilon+n}, \quad \text { i.e. } \quad S_{n} \stackrel{P}{\rightarrow} 0 \text { as } n \rightarrow \infty \text {. }
$$

Let

$$
T_{k}^{(1)}=a_{k}^{1} S_{1}+\ldots+a_{k}^{k} S_{k}=\frac{S_{1}+\ldots+S_{k}}{k},
$$


Now we prove that $T_{k}^{(1)} \stackrel{P}{\nrightarrow} 0$. Let $M_{k}=\sup \left(S_{1} \ldots S_{k}\right)$. Since $S_{i} \geq 0$, we have

which implies

$$
\frac{M_{k}}{k} \geq \varepsilon \Rightarrow \frac{S_{1}+\ldots+S_{k}}{k} \geq \varepsilon,
$$

$$
P\left(M_{k} / k \geq \varepsilon\right) \leq P\left(T_{k}^{(1)} \geq \varepsilon\right) .
$$

On the other hand,

$$
\begin{aligned}
P\left(M_{k}<x\right) & =P\left(S_{1}<x\right) \ldots P\left(S_{k}<x\right) \\
& =\left(1-\frac{1}{1+x}\right) \ldots\left(1-\frac{1}{k+x}\right)<\left(1-\frac{1}{k+x}\right)^{k} .
\end{aligned}
$$

It follows that

$$
P\left(M_{k} / k<\varepsilon\right)=P\left(M_{k}<k \varepsilon\right)<\left(1-\frac{1}{k \varepsilon+k}\right)^{k} .
$$

From (1) we conclude that

$$
P\left(T_{k}^{(1)} \geq \varepsilon\right) \geq 1-P\left(M_{k} / k<\varepsilon\right)>1-\left(1-\frac{1}{k \varepsilon+k}\right)^{k} .
$$

Finally, $\lim _{k \rightarrow \infty} P\left(T_{k}^{(1)} \geq \varepsilon\right) \geq 1-e^{-1 /(1+\varepsilon)} \neq 0$ and $T_{k}^{(1)} \stackrel{P}{\nrightarrow} 0$.

R e mark 3. Finally, concerning almost complete convergence, we give an example proving that we do not obtain a.c.-regularity.

As in the preceding example, take

$$
T_{k}^{(1)}=\frac{S_{1}+\ldots+S_{k}}{k}
$$

where $\left(S_{n}\right)$ is a sequence of independent random variables with distribution functions

$$
F_{n}(x)= \begin{cases}1-1 /\left(x+n^{2}\right) & \text { for } x>0 \\ 0 & \text { for } x \leq 0\end{cases}
$$

Then

$$
\forall \varepsilon>0, \quad \sum_{n \in \mathbb{N}^{*}} P\left(\left|S_{n}\right| \geq \varepsilon\right)=\sum_{n \in \mathbb{N}^{*}} \frac{1}{\varepsilon+n^{2}}<\infty .
$$

Hence $S_{n} \stackrel{\text { a.c. }}{\rightarrow} 0$. Let us prove that $T_{k}^{(1)} \stackrel{\text { a.c. }}{\leftrightarrow} 0$. Defining $M_{k}$ as in the preceding example, we have (1) and (2). It follows that

$$
P\left(M_{k}<x\right)<\left(1-\frac{1}{k^{2}+x}\right)^{k}
$$

and

$$
P\left(M_{k} / k<\varepsilon\right)=P\left(M_{k}<k \varepsilon\right)<\left(1-\frac{1}{k^{2}+k \varepsilon}\right)^{k}
$$


Hence

$$
P\left(T_{k}^{(1)} \geq \varepsilon\right) \geq 1-P\left(M_{k} / k<\varepsilon\right)>1-\left(1-\frac{1}{k^{2}+k \varepsilon}\right)^{k} .
$$

But

$$
1-\left(1-\frac{1}{k^{2}+k \varepsilon}\right)^{k}=1-e^{k L_{n}\left(1-\frac{1}{k^{2}+k \varepsilon}\right)} \underset{k \rightarrow \infty}{\sim} \frac{k}{k^{2}+k \varepsilon} \sim \frac{1}{k},
$$

which implies $\sum_{k=1}^{\infty} P\left(T_{k}^{(1)} \geq \varepsilon\right)=\infty$ and $T_{k}^{(1)} \stackrel{\underset{\text { a.c. }}{\nrightarrow}}{\rightarrow} 0$.

\section{References}

[1] P. Billingsley, Convergence of Probability Measures, Wiley, New York, 1968.

[2] D. Bosq et J. P. Lecoutre, Théorie de l'estimation fonctionnelle, Economica, Paris, 1987.

[3] C. Brezinski and M. Redivo Zaglia, Extrapolation Methods. Theory and Practice, North-Holland, Amsterdam, 1991.

[4] H. L. Gray, On a unification of bias reduction and numerical approximation, in: Probability and Statistics, J. N. Srivastance (ed.), North-Holland, Amsterdam, 1988, $105-116$.

[5] M. Ledoux and M. Talagrand, Probability in Banach Spaces, Springer, 1991.

[6] J. Neveu, Bases mathématiques du calcul des probabilités, Masson, 1964.

[7] V. Wimp, Sequence Transformations, Academic Press, New York, 1981.

HÉLÈNE LAVASTRE

LABORATOIRE DE STATISTIQUE ET PROBABILITÉS U.F.R. DE MATHÉMATIQUES PURES ET APPLIQUÉES 59655 VILLENEUVE D'ASCQ CEDEX, FRANCE 